\title{
Long non-coding RNA-guided regulation in organisms
}

\author{
QI WenChuan ${ }^{1}$, SONG Xu ${ }^{1,2 *} \&$ LI Ling $^{1 *}$ \\ ${ }^{1}$ Center for Functional Genomics and Bioinformatics, Key Laboratory of Bio-Resources and Eco-Environment of Ministry of Education, \\ College of Life Science, Sichuan University, Chengdu 610064, China; \\ ${ }^{2}$ State Key Laboratory of Biotherapy, West China Hospital, Sichuan University, Chengdu 610041, China
}

Received September 9, 2013; accepted September 16, 2013

\begin{abstract}
It is clear that RNA is more than just a messenger between gene and protein. The mammalian genome is pervasively transcribed, giving rise to tens of thousands of non-coding transcripts. Whether all of these transcripts are functional remains to be elucidated, but it is evident that there are many functional long non-coding RNAs (lncRNAs). Recent studies have set out to decode the regulatory role and functional diversity of lncRNAs. Here we organize these studies to highlight the significant involvements of IncRNAs in regulation of gene expression and human physiological and pathological processes, which are achieved by their interaction with DNA, RNA or protein.
\end{abstract}

IncRNAs, gene expression, physiological and pathological processes, interaction

Citation: Qi W C, Song X, Li L. Long non-coding RNA-guided regulation in organisms. Sci China Life Sci, 2013, 56: 891-896, doi: 10.1007/ s11427-013-4558-1

The great majority of mammalian genomes are potentially transcribed, the number of protein-coding genes, however, is fewer than initially anticipated and does not proportionally increase with the increased genome complexity $[1,2]$. Transcripts from the non-coding genome, which lack the evident protein-coding potential and function in the form of RNA molecules, are referred to as non-coding RNAs (ncRNAs). ncRNAs can be arbitrarily classified into small ncRNAs, typically 20-200 nt in length, and long ncRNAs (lncRNAs), ranging from $200 \mathrm{nt}$ to $>100 \mathrm{~kb}$ [3]. Much evidence on the significant role of small regulatory ncRNAs in gene expression has been widely accumulated [4], the research on lncRNAs, however, is still in a beginning stage.

Although the particular functions of lncRNAs still remain largely unclear, the appearance of their biological features indicates that IncRNAs, at least a sizable fraction of them, are not inert in function. For example, lncRNAs lacking strong sequence conservation are highly conserved

*Corresponding author (email: xusong@scu.edu.cn; lingli1980@scu.edu.cn) in their promoter regions; several lncRNAs are highly conserved in secondary structure and possess conservative splice sites; some lncRNAs are only expressed in specific stages of tissue differentiation and development or present apparent tissue-specific expression patterns and distinct subcellular localizations [5,6]. Furthermore, increasing evidence indicates that the dysregulation of lncRNAs is significantly involved in several types of diseases, including different types of cancer, Alzheimer's disease, Huntington's disease and cardiovascular diseases [7-11]. Altogether, we conclude that IncRNAs do not represent the "noise" of transcriptome, and the effort on decoding implication of lncRNAs in a variety of biological processes is expected to develop our knowledge on molecular biology to a newer frontier.

\section{Biological features of IncRNAs}

Many of the identified lncRNAs are transcribed by RNA 
polymerase II (RNAP II) and placed into the cluster of mRNA-like transcripts. This type of mRNA-like lncRNAs bear many signatures of mRNAs, including $5^{\prime}$ capping, splicing, and poly-adenylation, but possess little or no open reading frame $[12,13]$. While the abundance of lncRNAs has been demonstrated by large-scale cDNA sequencing projects, which identified more than 35000 lncRNAs from the transcripts across the human genome [14], certain subtypes of lncRNAs could be undervalued since the nonpolyadenylated transcripts have been omitted. As expected, the following data from tiling array indicated that more than $40 \%$ of transcripts are non-polyadenylated [15]. In addition, the identification of several RNAP III-produced lncRNAs further supports the opinion that the composition of lncRNAs is complicated. For instance, heat shock stimulates RNAP III to drive the expression of human Alu RNAs ( 300 nt) and mouse B2 RNA (217 nt) [16]. In contrast to abnormal physiology states that induce the confused expression of lncRNAs, the lower selective pressures under normal physiological conditions usually generate a strong cell type-specific expression of lncRNAs. In the latter case, the expression profiles of lncRNAs are generally more specific than that of protein-coding transcripts $[14,17,18]$.

To date, the origins of lncRNAs have been summarized as follows: (i) frame-disrupted transcription from proteincoding genes; (ii) chromosome recombination-facilitated locus throughout read; (iii) transposition effects from certain genetic elements such as retroposons; (iv) transcription of local tandem repeat sequences [19]. Based on the relative position with the protein-coding genes, IncRNAs could be further classified into the following five subtypes: (i) sense lncRNAs, sequences of which overlap with the sense strand of a protein-coding gene; (ii) antisense lncRNAs, which are expected to be transcribed from the antisense strand of a protein-coding gene; (iii) bidirectional lncRNAs, sequences of which are derived from the opposite strands of a protein-coding gene whose transcription start sites are located less than 1000 bp away; (iv) intronic lncRNAs, which are derived from the intronic region of a protein-coding gene; (v) intergenic lncRNAs, which are derived from the spacer of two separate protein-coding genes [19]. These classification rules are thought to facilitate the interpretation, as well as the further investigation, on lncRNAs.

Several studies have shown that specific lncRNAs are retained within the mammalian cell nucleus and are suggested to play structural roles or act as riboregulators $[18,20-24]$. The mammalian cell nucleus is compartmentalized into multiple non-membranous subnuclear domains such as nucleoli, nuclear speckles, paraspeckles, Cajal bodies and promyelocytic leukemia (PML) bodies, which in turn contain specific groups of proteins and RNAs involved in special nuclear processes $[25,26]$. With the respective identification of NEAT1 RNA and NEAT2 RNA in paraspeckles and splicing speckles, it has been further suggested that these nuclear-retained lncRNAs could play im- portant function in a variety of gene-regulatory processes observed in multicellular organisms, especially the transcriptional regulation and RNA metabolism [27].

In contrast to the small regulatory ncRNAs such as miRNAs and endo-siRNAs that regulate gene expression mainly through the direct base pairing to target sites within untranslated regions of mRNAs, lncRNAs achieve their regulatory function through quite diverse strategies. It has been reported that GAS5 RNA serves as a false target of transcription factor GR to prevent the binding of GR to the promoter DNA [28]; an IncRNA derived from the secondary promoter of $D H F R$ can bind in cis the primary promoter to form a DNA-RNA triplet, resulting in blocked recruitment of TFIID, a transcriptional regulation cofactor, to the primary promoter and the subsequent transcription repression [29]; linc-MD1 RNA acts as miRNA sponge to suppress the activities of miR-133 and miR-135, resulting in increased levels of miRNA targets and the ultimate influence on muscle differentiation [30]. In conclusion, the accumulated studies show that lncRNAs not only serve as the precursors of certain small ncRNAs, such as miR-675 encoded by the H19 IncRNA [24,31], but also present a class of regulatory RNAs fundamentally distinct from the small ncRNAs, which appear to play roles through the mechanism of interaction with DNA, RNA or protein.

\section{Biological functions of IncRNAs}

A large range of biological processes including dosage compensation, imprinting, cell division and cell differentiation are widely reported to be associated with IncRNAs, which are thought to work in cis on neighboring genes or in trans to regulate distantly located genes or molecular targets in nucleus and cytoplasm. In detail, IncRNAs can act as chromatin modifiers to influence the chromatin structure, as transcriptional regulators to affect the activities of transcription factors or RNA polymerase, as modular scaffolds to recruit the assembly of multiple protein complexes, and as "false decoys" to sponge the coupled targets and thereby inhibit their correct cellular localization [3,19,32-37]. Altogether, the current studies suggest that lncRNAs could be involved in almost each step of gene expression, such as epigenetic regulation, transcriptional regulation and posttranscriptional regulation, through in cis or in trans manner, and the dysregulation of IncRNAs would cause broad changes in cell signaling pathways.

\subsection{IncRNAs in regulation of gene expression}

\subsubsection{Epigenetic regulation}

Epigenetic regulation of gene expression, which is characterized as the altered transcription without any change in gene sequence, is generally reported to play roles in organism development as well as tumorigenesis. Recently, the 
involvement of lncRNAs in epigenetic regulation has been widely documented. Kcnq1ot1, a nuclear-retained lncRNA with the length of $91.5 \mathrm{~kb}$, is transcribed by RNAPII from the intron 10 of Kcnql gene in an antisense orientation to Kcnq1 [38]. The production of Kcnq1ot1 RNA was first found to be associated with the lineage-specific silencing of dozens of genes within the Kcnql locus [38,39]. Recent studies further indicated that the silencing effects are achieved by the interactions of Kcnq1ot1 with chromatin and with the H3K9- and H3K27-specific histone methyltransferases G9a and the polycomb repressive complex 2 (PRC2) [40]. HOTAIR and Air are the other two well-characterized lncRNAs involved in chromatin remodeling, which exert function through a similar fashion, including accumulation at the chromatin regions of silenced alleles and the subsequent mediation of repressive histone modification through recruiting the specific chromatin modification complexes such as G9a, PRC1 and PRC2 [41-44]. Interestingly, another group reports that Kcnq1ot1 RNA is also required for the maintenance of the silencing of ubiquitously imprinted genes (UIGs) through guiding and maintaining the $\mathrm{CpG}$ methylation at methylated regions flanking the UIGs [45]. Although the crosstalk between lncRNA-mediated DNA methylation and lncRNA-mediated histone modification remains to be elucidated, these studies highlight the significant involvement of lncRNAs in epigenetic regulation of gene expression.

\subsubsection{Transcriptional regulation}

Transcription is a tightly regulated process in eukaryotes. In addition to the well-known factors such as RNA polymerase, general transcription factors and gene-specific transcription factors, it is also suggested that lncRNAs function in the complicated regulatory network to make gene expression more symphonic. Thus, a current central issue is to obtain a full understanding of the potential role of lncRNAs in regulated gene transcription programs, possibly through diverse mechanisms.

Through recruiting and modulating the activities of co-regulators, lncRNAs may act as selective ligands to prevent the transcription of target genes. Wang et al. [46] reported that DNA damage induces the production of several lncRNAs from the $5^{\prime}$ regulatory region of cyclin D1 $(C C N D 1)$. These induced lncRNAs specifically bind and allosterically modify TLS, a regulatory sensor of DNA damage, leading to the interaction of the modified TLS with CREB-binding protein (CBP) that thereby inhibits the transcription of CCND1. Martianov et al. [29] reported another example of inhibitory IncRNA that is induced by serum starvation and functions as a promoter-specific transcriptional repressor. Upon transcribed from the upstream minor promoter of DHFR gene, this lncRNA not only binds transcription factor II B (TFIIB) to prevent its association with the major promoter, but also forms a stable complex with the major promoter that interferes in the promoter-directed recruitment of TFIIB.

In addition to the inhibitory effect on transcription, lncRNAs could also act as activators or co-activators to upregulate gene expression. For instance, steroid receptor RNA activator (SRA) is an lncRNA transcript that acts as a eukaryotic transcriptional co-activator for steroid hormone receptors [47]. Another IncRNA, Evf-2, which is transcribed from one of the two Dlx-5/6 conserved intergenic regions, activates transcriptional activity of homeodomain proteins by directly influencing Dlx-2 activity [48]. The functional importance of gene enhancers in regulated gene expression has been well established, and the subsequent identification of bidirectional lncRNAs transcribed from enhancers, termed as enhancer RNAs (eRNAs), adds another functional layer to the transcriptional regulatory elements [49-51]. A subtype of eRNAs, which are derived from enhancers adjacent to E2-upregulated coding genes, were observed to contribute to E2-dependent gene activation by stabilizing E2/ER- $\alpha$ /eRNA-induced enhancer-promoter looping, suggesting that eRNAs are functional but not merely a reflection of enhancer activation [52].

\subsubsection{Post-transcriptional regulation}

One of the most versatile processing steps in the life of an mRNA molecule is pre-mRNA splicing, which provides a major source of transcriptome and proteome diversity in cells [53]. Although the spliceosome is required to perform splicing catalysis and additional splicing factors function in determining the splice sites, several recent studies have highlighted the significant role of lncRNAs in the regulation of pre-mRNA splicing. Depletion of MALAT1 RNA is reported to compromise the recruitment of SR proteins from speckles to the sites of transcription, where splicing occurs [54]. Meanwhile, Tripathi et al. [55] indicate that MALAT1 could act as a "molecular sponge" by interacting with SR proteins, especially SRSF1, in the nuclear speckles, and thereby modulate the concentration of splicing-competent SR proteins in cells. This "sponge" mechanism is also applicable to the untranslated region (UTR) of certain protein-coding genes. For instance, the repeat-containing RNA from the CTG expansion in 3'UTR of DMPK gene accumulates in the nucleus and affects the activity of splicing factors. Similarly, CGG repeats occurred in FMRI gene can recruit a set of splicing regulators into nuclear inclusions [56].

miRNAs function as important post-transcriptional regulators by triggering mRNA degradation or translational inhibition. Recently, miRNA activity has been proposed to be affected by some endogenous sponge transcripts that contain the miRNA-matching sequence. For instance, in human and mouse brain, a highly expressed circular lncRNA, termed as ciRS-7, is reported to act as a miR-7 sponge. The sponge effect is mediated by the selectively conserved miRNA target sites contained in ciRS-7, which strongly suppress miR-7 activity and thereby result in increased lev- 
els of miR-7 targets [57]. In addition, identification of additional sponge lncRNAs, such as testis-specific Sry that targets miR-138, suggests that miRNA sponge effects could be a general phenomenon in organisms [30,58-62].

Furthermore, IncRNAs may also apply regulatory pressure to other aspects of post-transcriptional regulation, such as RNA metabolism and translation [63-66].

\subsection{IncRNAs in tumorigenesis}

Comparison of gene expression profiles of tumor and normal cells has revealed a linkage of lncRNAs with tumorigenesis. For example, OCC-1 RNA is overexpressed in colon cancer cells [67], and the overexpression of lncRNA PCEGEM1 could induce proliferation of cancer cells [68]. MALAT1 RNA is another documented metastasis-associated IncRNA, whose overexpression is associated with nonsmall cell lung cancer, breast cancer, colon cancer, prostatic cancer and liver cancer [69].

Similar to miRNAs, IncRNAs can also serve as the potential molecular targets for diagnosis and treatment of cancer. However, there are only limited studies decoding the detailed role of IncRNAs in tumorigenesis. Based on the ability to repress proto-oncogenes, PSF functions as a tumor-suppressor protein, whose oncogenesis suppression activity, however, can be impaired by the enhancement of its RNA binding ability [70,71]. The subsequent studies identify several PSF-binding lncRNAs from mice and humans, which include retroposon-derived lncRNAs such as VL30 and frame-disrupted non-coding transcripts such as MALAT1. All the lncRNAs promote tumorigenesis in mice and humans through a mechanism of reversible regulation on proto-oncogene transcription, including the protein PSF that binds to the regulatory region of a proto-oncogene and represses transcription, and PSF-binding lncRNA that binds to PSF, forming a PSF-lncRNA complex that dissociates from a proto-oncogene, activating transcription [72-76]. ANRIL and HOTAIR are two other tumorigenesis-related IncRNAs, which are discovered to recruit the PRC complex to locus in cis or specific target genes genome-wide, leading to histone methylation, epigenetic silencing of metastasis suppressor genes and increased tumor invasiveness and metastasis $[43,44,77]$.

p53 is considered to be one of the most common denominators in human cancer and plas a central role in regulatory networks responsible for cancer-related stress [78-80]. It seems controversial to explain p53 pathway only from the perspective of protein-coding genes [81-83]. As expected, a large number of miRNAs and conserved miRNA families are found to be direct transcriptional targets of p53 or function as the upstream regulators of p53 [84]. Several current studies further illustrate the linkage between lncRNAs and p53 pathway from the perspective of molecular biology. p53-activated lincRNA-p21 serves as a repressor, through interacting with heterogeneous nuclear ribonucleoprotein $\mathrm{K}$
(hnRNP-K), in p53-dependent transcriptional response. Inhibition of lincRNA-p21 results in a global change in expression of hundreds of gene targets, a majority of which are downstream targets repressed by p53 and responsible for p53-mediated apoptosis [85]. Upon induced by p53, PANDA RNA interacts with transcription factor NF-YA to inhibit expression of proapoptosis genes, leading to cell cycle arrest [86]. Another study reports lncRNA RoR acts as an upstream p53 repressor in response to DNA damage. Unlike MDM2 that causes p53 degradation through the ubiquitin-proteasome pathway, RoR suppresses p53 translation through directly interacting with the hnRNP-I [87]. Collectively, these data reinforces the growing awareness that epigenetic regulation plays significant roles in tumor development by placing lncRNAs in the heartland of a well-known tumor-regulation network.

\subsection{IncRNAs in immune response and host defense}

Expression pattern of lncRNAs in cells is influenced by virus infection and lipopolysaccharide (LPS) stimulation $[5,88]$. NeST is the first lncRNA discovered in the immune system, which was showed to control susceptibility to Theiler's virus and Salmonella infection in mice through epigenetic regulation of the IFN- $\gamma$ locus $[89,90]$. The innate immune system coordinates host defenses through germ line-encoded pattern recognition receptors such as Toll-like receptors (TLRs), which recognize microbial products and induce the expression of hundreds of protein-coding genes involved in antimicrobial defense and adaptive immunity [91]. A recent study reveals that Toll-like receptors induce the expression of numerous IncRNAs. One of these, lincRNA-Cox2, can mediate both activation and repression of distinct classes of immune genes, and the repressive effect is dependent on interactions of lincRNA-Cox 2 with hnRNP-A/B and hnRNP-A2/B1 [92]. Collectively, these reports unveil a central role of lncRNAs as broad-acting regulators in immune response and host defense.

\section{Conclusion}

Results from large-scale analyses of transcriptome propose that tens of thousands of IncRNA are pervasively transcribed from mammalian genomes. Moreover, even in some simple multicellular organisms, the amount of lncRNAs is far greater than that of the total small ncRNAs. We are only beginning to understand the realm of IncRNAs, whose representation in the genome, according to comparative analyses, increases with the increased genome complexity. The discovery that lncRNAs are central to numerous pivotal biological processes may reflect ancient connections between IncRNAs and the regulation of developmental and physiological decisions, whose disruption can lead to physiological disorders as well as several types of diseases. 
Some lncRNAs affect a great number of downstream targets. This raises a possibility that the adoption of lncRNAs permits pathways to simultaneously regulate many aspects of a particular cellular process.

This work was supported by grants from the National Basic Research Program of China (2011CB504203), National Natural Science Foundation of China (31000579, 31371325), Innovative Research Team Program of Sichuan Province (2011JTD0026), and the Program for New Century Excellent Talents in the University of Ministry of Education of China (NCET-10-0599).

1 Amaral P P, Dinger M E, Mercer T R, et al. The eukaryotic genome as an RNA machine. Science, 2008, 319: 1787-1789

2 Mattick J S. The genetic signatures of noncoding RNAs. PLoS Genet, 2009, 5: e1000459

3 Mercer T R, Dinger M E, Mattick J S. Long non-coding RNAs: Insights into functions. Nat Rev Genet, 2009, 10: 155-159

4 Carthew R W, Sontheimer E J. Origins and mechanisms of miRNAs and siRNAs. Cell, 2009, 136: 642-655

5 Guttman M, Amit I, Garber M, et al. Chromatin signature reveals over a thousand highly conserved large non-coding RNAs in mammals. Nature, 2009, 458: 223-227

6 Ulitsky I, Shkumatava A, Jan C H, et al. Conserved function of lincRNAs in vertebrate embryonic development despite rapid sequence evolution. Cell, 2011, 147: 1537-1550

7 Spizzo R, Almeida M I, Colombatti A, et al. Long non-coding RNAs and cancer: A new frontier of translational research? Oncogene, 2012 31: 4577-4587

8 Tsai M C, Spitale R C, Chang H Y. Long intergenic noncoding RNAs: New links in cancer progression. Cancer Res, 2011, 71: 3-7

9 McPherson R, Pertsemlidis A, Kavaslar N, et al. A common allele on chromosome 9 associated with coronary heart disease. Science, 2007, 316: 1488-1891

10 Johnson R. Long non-coding RNAs in Huntington's disease neurodegeneration. Neurobiol Dis, 2012, 46: 245-254

11 Tan L, Yu J T, Hu N, et al. Non-coding RNAs in Alzheimer's disease. Mol Neurobiol, 2013, 47: 382-393

12 Du Toit A. Non-coding RNA: RNA stability control by Pol II. Nat Rev Mol Cell Biol, 2013, 14: 128

13 Carninci P, Kasukawa T, Katayama S, et al. The transcriptional landscape of the mammalian genome. Science, 2005, 309: 15591563

14 Djebali S, Davis C A, Merkel A, et al. Landscape of transcription in human cells. Nature, 2012, 489: 101-108

15 Cheng J, Kapranov P, Drenkow, J, et al. Transcriptional maps of 10 human chromosomes at 5-nucleotide resolution. Science, 2005, 308: 1149-1154

16 Goodrich J A, Kugel J F. Dampening DNA binding: A common mechanism of transcriptional repression for both ncRNAs and protein domains. RNA Biol, 2010, 7: 305-309

17 Ravasi T, Ruzuki H, Pang K C, et al. Experimental validation of the regulated expression of large numbers of non-coding RNAs from the mouse genome. Genome Res, 2006, 16: 11-19

18 Batista P J, Chang H Y. Long noncoding RNAs: Cellular address codes in development and disease. Cell, 2013, 152: 1298-1307

19 Ponting C P, Oliver P L, Reik W. Evolution and functions of long noncoding RNAs. Cell, 2009, 136: 629-641

20 Prasanth K V, Spector D L. Eukaryotic regulatory RNAs: An answer to the "genome complexity" conundrum. Gene Dev, 2007, 21: 11-42

21 Wilusz J E, Sunwoo H, Spector D L. Long noncoding RNAs: Functional surprises from the RNA world. Genes Dev, 2009, 23: 1494-1504

22 Cremer T, Kupper K, Dietzel S, et al. Higher order chromatin architecture in cell nucleus: On the way from structure to function. Biol Cell, 2004, 96: 555-567
23 Fox A H, Lamond A I. Paraspeckles. Cold Spring Harb Perspect Biol, 2010, 2: a000687

24 Kapranov P, Cheng J, Dike S, et al. RNA maps reveal new RNA classes and a possible function for pervasive transcription. Science, 2007, 316: 1484-1488

25 Matera A G, lzaguire-Sierra M, Praveen K, et al. Nuclear bodies: Random aggregates of sticky proteins or crucibles of macromolecular assembly? Dev Cell, 2009, 17: 639-647

26 Spector D L. SnapShot: Cellular bodies. Cell, 2006, 127: 1071

27 Cautron-Herger M, Rippe K. Nuclear architecture by RNA. Curr Opin Genet Dev, 2012, 22: 179-187

28 Kino T, Hurt D E, Ichijo T, et al. Noncoding RNA gas5 is a growth arrest- and starvation-associated repressor of the glucocorticoid receptor. Sci Signal, 2010, 3: ra8

29 Martianov I, Ramadass A, Serra Barros A, et al. Repression of the human dihydrofolate reductase gene by a non-coding interfering transcript. Nature, 2007, 445: 666-670

30 Cesana M, Cacchiarelli D, Legnini I, et al. A long noncoding RNA controls muscle differetiation by functioning as a competing endogenous RNA. Cell, 2011, 147: 358-369

31 Keniry A, Oxley D, Monnier P, et al. The H19 lincRNA is a developmental reservoir of miR-675 that suppresses growth and lgf1r. Nat Cell Biol, 2012, 14: 659-665

32 Guil S, Esteller M. Cis-acting noncoding RNAs: Friends and foes. Nat Struct Mol Biol, 2012, 19: 1068-1075

33 Wang K C, Chang H Y. Molecular mechanisms of long noncoding RNAs. Mol Cell, 2011, 43: 904-914

34 Lee J T. Epigenetic regulation by long noncoding RNAs. Science, 2012, 338: 1435-1439

35 Rinn J L, Chang H Y. Genome regulation by long noncoding RNAs. Annu Rev Biochem, 2012, 81: 145-166

36 Kelley R L, Meller V H, Gordadze P R, et al. Epigenetic spreading of the Drosophila dosage compensation complex from roX RNA genes into flanking chromatin. Cell, 1999, 98: 513-522

37 Koziol M J, Rinn J L. RNA traffic control of chromatin complexes. Curr Opin Genet Dev, 2010, 20: 142-148

38 Mancini-Dinardo D, Steele S J, Levorse J M. Elongation of Kcnq1ot1 transcript is required for genomic imprinting of neighboring genes. Genes Dev, 2006, 20: 1268-1282

39 Fitzpatrick G V, Soloway P D, Higgins M J. Regional loss of imprinting and growth deficiency in mice with a targeted deletion of KvDMR1. Nat Genet, 2002, 32: 426-431

40 Pandey R R, Mondal T, Mohammad F, et al. Kcnq1ot1 antisense noncoding RNA mediates lineage-specific transcriptional silencing through chromatin-level regulation. Mol Cell, 2008, 32: 232-246

41 Rinn J L, Kertesz M, Wang J K, et al. Functional demarcation of active and silent chromatin domains in human HOX loci by noncoding RNAs. Cell, 2007, 129: 1311-1323

42 Nagano T, Mitchell J A, Sanz L A, et al. The Air noncoding RNA epigenetically silences transcription by targeting G9a to chromatin. Science, 2008, 322: 1717-1720

43 Tsai M C, Manor O, Wan Y, et al. Long noncoding RNA as modular scaffold of histone modification complexes. Science, 2010, 329: 689-693

44 Gupta R A, Shah N, Wang K C, et al. Long non-coding RNA HOTAIR reprograms chromatin state to promote cancer metastasis. Nature, 2010, 464: 1071-1076

45 Mohammad F, Pandey G K, Mondal T, et al. Long noncoding RNA-mediated maintenance of DNA methylation and transcriptional gene silencing. Development, 2012, 139: 2792-2803

46 Wang X, Arai S, Song X, et al. Induced ncRNAs allosterically modify RNA-binding proteins in cis to inhibit transcription. Nature, 2008, 454: $126-130$

47 Lanz R B, Razani B, Goldberg A D, et al. Distinct RNA motifs are important for coactivation of steroid hormone receptors by steroid receptor RNA activator (SRA). Proc Natl Acad Sci USA, 2002, 99: 16081-16086

48 Feng J, Bi C, Clark B S, et al. The Evf-2 noncoding RNA is transcribed from the Dlx-5/6 ultraconserved region and functions as a 
Dlx-2 transcriptional coactivator. Genes Dev, 2006, 20: 1470-1484

49 Kim T K, Hemberg M, Gray J M, et al. Widespread transcription at neuronal activity-regulated enhancers. Nature, 2010, 465: 182-187

50 Hah N, Danko C G, Core L, et al. A rapid, extensive, and transient transcriptional response to estrogen signaling in breast cancer cells. Cell, 2011, 145: 622-634

51 Wang D, Garcia-Bassets I, Benner C, et al. Reprogramming transcription by distinct classes of enhancers functionally defined by eRNA. Nature, 2011, 474: 390-394

52 Li W, Notani D, Ma Q, et al. Functional roles of enhancer RNAs for oestrogen-dependent transcriptional activation. Nature, 2013, 498: 516-520

53 Pan Q, Shai O, Lee L J, et al. Deep surveying of alternative splicing complexity in the human transcriptome by high-throughput sequencing. Nat Genet, 2008, 40: 1413-1415

54 Bernard D, Prasanth K V, Tripathi V, et al. A long nuclear-retained non-coding RNA regulates synaptogenesis by modulating gene expression. EMBO J, 2010, 29: 3082-3093

55 Tripathi V, Ellis J D, Shen Z, et al. The nuclear-retained noncoding RNA MALAT1 regulates alternative splicing by modulating SR splicing factor phosphorylation. Mol Cell, 2010, 39: 925-938

56 Sellier C, Rau F, Liu Y, et al. Sam68 sequestration and partial loss of function are associated with splicing alterations in FXTAS patients. EMBO J, 2010, 29: 1248-1261

57 Hansen T B, Jensen T I, Clausen B H, et al. Natural RNA circles function as efficient microRNA sponges. Nature, 2013, 495: 384-388

58 Franco-Zorrilla J M, Valli A, Todesco M, et al. Target mimicry provides a new mechanism for regulation of microRNA activity. Nat Genet, 2007, 39: 1033-1037

59 Poliseno L, Salmena L, Zhang J, et al. A coding-independent function of gene and pseudogene mRNAs regulates tumour biology. Nature, 2010, 465: 1033-1038

60 Karreth F A, Tay Y, Perna D, et al. In vivo identification of tumor-suppressive PTEN ceRNAs in an oncogenic BRAF-induced mouse model of melanoma. Cell, 2011, 147: 382-395

61 Tay Y, Kats L, Salmena L, et al. Coding-independent regulation of the tumor suppressor PTEN by competing endogenous mRNAs. Cell, 2011, 147: 344-357

62 Sumazin $\mathrm{P}$, Yang $\mathrm{X}$, Chiu $\mathrm{H} \mathrm{S}$, et al. An extensive microRNA-mediated network of RNA-RNA interactions regulates established oncogenic pathways in glioblastoma. Cell, 2011, 147: 370-381

63 Wang H, Iacoangeli A, Lin D, et al. Dendritic BC1 RNA in translational control mechanisms. J Cell Biol, 2005, 171: 811-821

64 Centonze D, Rossi S, Napoli I, et al. The brain cytoplasmic RNA $\mathrm{BC} 1$ regulates dopamine $\mathrm{D} 2$ receptor-mediated transmission in the striatum. J Neurosci, 2007, 27: 8885-8892

65 Lewejohann L, Skryabin B V, Sachser N, et al. Role of a neuronal small non-messenger RNA: Behavioural alterations in BC1 RNA-deleted mice. Behav Brain Res, 2004, 154: 273-289

66 Gong C, Maquat L E. IncRNAs transactivate stau1-mediated mRNA decay by duplexing with $3^{\prime}$ UTRs via Alu elements. Nature, 2011, 470: 284-288

67 Pibouin L, Villaudy J, Ferbus D, et al. Cloning of the mRNA of overexpression in colon carcinoma-1: A sequence overexpressed in a subset of colon carcinomas. Cancer Genet Cytogenet, 2002, 133: 55-60

68 Fu X, Ravindranath L, Tran N, et al. Regulation of apoptosis by a prostate-specific and prostate cancer-associated noncoding gene, PCGEM1. DNA Cell Biol, 2006, 25: 135-141

69 Ji P, Diederichs S, Wang W, et al. MALAT-1, a novel noncoding RNA, and thymosin beta4 predict metastasis and survival in earlystage non-small cell lung cancer. Oncogene, 2003, 22: 8031-8041

70 Galietta A, Gunby R H, Redaelli S, et al. NPM/ALK binds and phosphorylates the RNA/DNA-binding protein PSF in anaplastic large-cell lymphoma. Blood, 2007, 100: 2600-2609
71 Figueroa A, Fujita Y, Gorospe M. Hacking RNA: Hakai promotes tumorigenesis by enhancing the RNA-binding function of PSF. Cell Cycle, 2009, 8: 3648-3651

72 Song X, Wang B, Bromberg M, et al. Retroviral-mediated transmission of a mouse VL30 RNA to human melanoma cells promotes metastasis in an immunodeficient mouse model. Proc Natl Acad Sci USA, 2002, 99: 6269-6273

73 Song X, Sui A, Garen A. Binding of mouse VL30 retrotransposon RNA to PSF protein induces genes repressed by PSF: Effects on steroidogenesis and oncogenesis. Proc Natl Acad Sci USA, 2004, 101: 621-626

74 Song X, Sun Y, Garen A. Roles of PSF protein and VL30 RNA in reversible gene regulation. Proc Natl Acad Sci USA, 2005, 102: 12189-12193

75 Wang G, Cui Y, Zhang G, et al. Regulation of proto-oncogene transcription, cell proliferation, and tumorigenesis in mice by PSF protein and a VL30 noncoding RNA. Proc Natl Acad Sci USA, 2009, 106: 16794-16798

76 Li L, Feng T, Lian Y, et al. Role of human noncoding RNAs in the control of tumorigenesis. Proc Natl Acad Sci USA, 2009, 106: 16794-16798

77 Yap K L, Munoz-Cabello A M, Raguz S, et al. Molecular interplay of the noncoding RNA ANRIL and methylated histone $\mathrm{H} 3$ lysine 27 by polycomb CBX7 in transcriptional silencing of INK4a. Mol Cell, 2010, 38: 662-674

78 Lane D P, Fischer P M. Turning the key on p53. Nature, 2004, 427: 789-790

79 Lowe S W, Cepero E, Evan G. Intrinsic tumour suppression. Nature, 2004, 432: 307-315

80 Vogelstein B B, Lane D D, Levine A A. Surfing the p53 network. Nature, 2000, 408: 307-310

81 Yu J, Zhang L, Hwang P M, et al. Identification and classification of p53-regulated genes. Proc Natl Acad Sci USA, 1999, 96: 14517-14522

82 Zhao R, Gish K, Mruphy M, et al. Analysis of p53-regualted gene expression patterns using oligonucleotide arrays. Genes Dev, 2000, 14: 981-993

83 Brugarolas J, Chandrasekaran C, Gordon J I, et al. Radiation-induced cell cycle arrest compromised by p21 deficiency. Nature, 1995, 337: 552-557

84 Suzuki H I, Miyazono K. Dynamics of microRNA biogenesis: Crosstalk between p53 network and microRNA processing pathway. J Mol Med (Berl), 2010, 88: 1085-1094

85 Huarte M, Guttman M, Feldser D, et al. A large intergenic noncoding RNA induced by p53 mediates global gene repression in the p53 response. Cell, 2010, 142: 409-419

86 Hung T, Wang Y, Lin M F, et al. Extensive and coordinated transcription of noncoding RNAs within cell-cycle promoters. Nat Genet, 2011, 43: 621-629

87 Zhang A, Zhou N, Huang J, et al. The human long non-coding RNA-RoR is a p53 repressor in response to DNA damage. Cell Res, 2013, 23: 340-350

88 Peng X, Gralinski L, Armour C D, et al. Unique signatures of long noncoding RNA expression in response to virus infection and altered innate immune signaling. MBio, 2010, 1: e00206-e0210

89 Collier S P, Collins P L, Williams C L, et al. Cutting edge: Influence of Tmevpg1, a long intergenic noncoding RNA, on the expression of lfng by Th1 cells. J Immunol, 2012, 189: 2084-2088

90 Gomez J A, Wapinski O L, Yang Y W, et al. The NeST long ncRNA controls microbial susceptibility and epigenetic activation of the interferon- $\gamma$ locus. Cell, 2013, 152: 743-754

91 Medzhitov R, Horng T. Transcriptional control of the inflammatory response. Nat Rev Immunol, 2009, 9: 692-703

92 Carpenter S, Aiello D, Atianand M K, et al. A long noncoding RNA mediates both activation and repression of immune response genes. Science, 2013, 341: 789-792

Open Access This article is distributed under the terms of the Creative Commons Attribution License which permits any use, distribution, and reproduction in any medium, provided the original author(s) and source are credited. 